Center for

Mathematical Economics

Working Papers

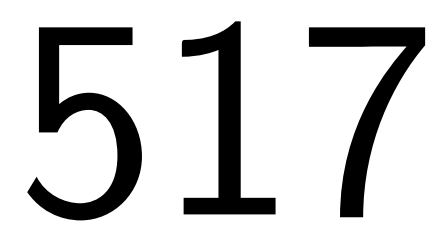

August 2014

\title{
Stable Networks in Homogeneous Societies
}

Tim Hellmann and Jakob Landwehr

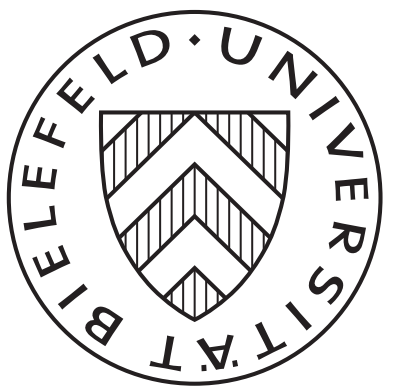

Center for Mathematical Economics (IMW)

Bielefeld University

Universitätsstraße 25

D-33615 Bielefeld · Germany

e-mail: imw@uni-bielefeld.de 


\title{
Stable Networks in Homogeneous Societies
}

\author{
Tim Hellmann* and Jakob Landwehr ${ }^{\dagger}$
}

August 7, 2014

\begin{abstract}
We study the structure of pairwise stable networks from a very general point. Rather than assuming a particular functional form of utility, we simply assume that the society is homogeneous, i.e. that agents' utilities differ only with respect to their network position while their names do not matter. Existence of certain stable network structures is then implied by fairly general assumptions on externalities between links. Depending on the form of link externalities, either the empty or complete network are always pairwise stable, stable symmetric networks exist, or stable networks with a connected subgroup exist. If the society becomes more homogeneous, then it is possible to characterize the set of all pairwise stable networks: they are nested split graphs (NSG). We illustrate these results with many examples from the literature, including utility profiles that depend on centrality measures such as Bonacich centrality. In particular, for low discount factors every pairwise stable network is an NSG if utility is given by Bonacich centrality.
\end{abstract}

Keywords: Network Formation, Pairwise Stability, Existence, Homogeneity, Convexity, Strategic Complements, Bonacich Centrality

JEL-Classification: A14, C72, D85.

\footnotetext{
${ }^{*}$ Center for Mathematical Economics, Bielefeld University, postbox 100131, D-33501 Bielefeld, Germany. Email: tim.hellmann@uni-bielefeld.de, phone: +49 521106 2573, fax: +49 521 1062997.

${ }^{\dagger}$ Center for Mathematical Economics, Bielefeld University, postbox 100131, D-33501 Bielefeld, Germany. Email: jakob.landwehr@uni-bielefeld.de, phone: +49521 1064918
} 


\section{Introduction}

Starting with the seminal contribution of Jackson and Wolinsky (1996), a substantial literature has evolved modeling strategic network formation. Economic agents in these models have a preference ordering over the set of networks. Examples include firms' profit when forming R\&D networks (Goyal and Joshi, 2003), countries' social welfare when forming trade agreements (Goyal and Joshi, 2006a), and individuals' importance when forming friendships (Jackson and Wolinsky, 1996). Since the structure of interaction, i.e. the social network, affects everydays economic outcomes, it is interesting to economists which kind of interaction structures emerge when links are formed strategically. The seminal concept of such equilibrium outcomes is the notion of pairwise stability (Jackson and Wolinsky, 1996). A central question is then under which conditions stable networks exist and which structure they have.

In this paper, we approach this question from a very general point. Rather than assuming a particular functional form of utility, we simply assume a homogeneous society where each agent's utility depends only on her network position but not on her name. We then show that some ordinal link externality conditions on the utility function are sufficient for the existence of stable networks of particular architecture. These ordinal link externality conditions define solely the impact that new links have on incentives to form own links, like ordinal convexity, i.e. ordinal increasing returns in own links, and ordinal strategic complements, i.e. ordinal increasing incentives to form own links in other agents' links. We show that if one of these link externalities on marginal utility is positive then pairwise stable networks of certain structure exists. Which class of networks arise as stable depends on which externality property is satisfied (see Propositions 1, 3, and 4).

While these link externality properties guarantee existence, they are not sufficient to characterize classes of networks which contain all pairwise stable networks. To achieve that, we impose stronger assumptions on the homogeneity of the society in combination with the link externality properties. These stronger conditions are expressing a general desire to be central in the network which is commonly observed in network formation models starting with Jackson and Wolinsky (1996). We show that with these stronger notions of positive link externalities all pairwise stable networks are contained in the class of nested split graphs (Proposition 2). Nested split graphs (Cvetković and Rowlinson, 1990) are networks where the set of neighbors of any two players can be ordered according to the set inclusion ordering. As the society becomes more and more homogeneous, then the pairwise stable networks are only found in subclasses of the nested split graphs, the so called dominant group networks (Propositions 5 and 6).

We illustrate our general results with respect to several important applications. 
Among those is a model of network formation such that the utility of players is given by their Bonacich centrality (Bonacich, 1987). Such a utility function arises e.g. when individuals form costly links in the first stage and then engage in team production in the second stage. Indeed, Ballester et al. (2006) show that the unique pure strategy equilibrium of the second stage in such a game is determined by the Bonacich centrality. This measure of centrality counts the number of paths emanating from a given node which are discounted by the length of each path with a common discount factor. Utility functions given by Bonacich centrality give rise to the positive link externalities and, even more interestingly, for small discount factors, also our stronger link externality properties are satisfied. Hence applying our general results to utility given by Bonacich centrality, we can conclude that either the empty network or the complete network are necessarily pairwise stable (for any discount factor), while any pairwise stable network is of nested split structure, respectively dominant group structure if the discount factor is small enough.

General properties of stable networks are of high interest for several reasons. Our results may help characterize stable networks for future (maybe very complex) models of network formation, and they provide reasoning why certain stability structures emerge in existing models of network formation: the driving force are the link externality conditions. That our results are applicable to so many settings is due to the generality our approach and the fact that the assumption of a homogeneous society is not restrictive as almost all models of strategic network formation share this property (cf. e.g. several surveys and textbooks including Jackson, 2003, 2006; Goyal, 2005, 2007; Vega-Redondo, 2007; Jackson, 2008; Easly and Kleinberg, 2010; Hellmann and Staudigl, 2014).

Although the literature on strategic network formation is enormous, only few results concerning these general structural properties can be found. Exceptions are Jackson and Watts (2001) and Chakrabarti and Gilles (2007) who use the restrictive assumption of a potential function (Monderer and Shapley, 1996) to prove existence of stable networks, and the recent paper Hellmann (2013) who similar to our approach - uses link externality conditions to show existence and uniqueness of stable networks. In light of their general approach, these papers, however, are not able to show existence of particular stable networks. We fill this gap with the help of the homogeneity assumption.

Assuming more structure on the functional form of utility, Goyal and Joshi (2006b) are also able to show existence of particular stable network structures such as regular networks, dominant group structures, and exclusive group structures depending on cardinal link externalities. ${ }^{1}$ They, however, assume a specific form of utility depending only on a particular network statistic, the vector of

\footnotetext{
${ }^{1}$ Regular networks are such that all nodes have the same number of neighbors (degree), while we refer the reader to Goyal and Joshi (2006b) for a definition of exclusive group structures.
} 
agents' degrees. We show that some of their results can be generalized in two ways: first, they hold for arbitrary utility functions in a homogeneous society; second, the link externality conditions can be generalized to hold also in ordinal terms. Thereby, our results are applicable to many examples of utility which are not captured in the framework of Goyal and Joshi (2006b), Jackson and Watts (2001) and Chakrabarti and Gilles (2007). In these examples, our results contribute substantially more than the more general setup in Hellmann (2013). Among those is the afore mentioned utility function given by Bonacich centrality.

The rest of the paper is organized as follows. Section 2 defines the model and presents the important assumptions and definitions used throughout the paper. Section 3 presents the results ordered by the externalities that are respectively assumed. Section 5 concludes.

\section{The model}

Let $N=\{1,2, \ldots, n\}$ be a finite set of agents. Depending on the application these can be firms, countries, individuals, etc. These economic agents strategically form links and, thus, are henceforth called players. Throughout this paper we will assume network formation to be undirected. A connection or link between two players $i \in N$ and $j \in N, i \neq j$ will be denoted by $\{i, j\}$ which we abbreviate for simplicity by $i j=j i:=\{i, j\}$. We then define the complete network $g^{N}=$ $\{i j \mid i, j \in N, i \neq j\}$ as the network where any two players are connected to each other and the set of all networks $G=\left\{g \mid g \subseteq g^{N}\right\}$.

We will further denote the set of links of some player $i$ in a network $g$ by $L_{i}(g)=$ $\{i j \in g \mid j \in N\}$, and all other links $L_{-i}(g)=g-L_{i}(g)$ where $g-g^{\prime}:=g \backslash g^{\prime}$ denotes the network obtained by deleting the set of links $g^{\prime} \cap g$ from network $g$. Analogously, $g+g^{\prime}:=g \cup g^{\prime}$. The set of player $i^{\prime}$ s neighbors is given by $N_{i}(g)=\{j \in N \mid i j \in g\}$ and $\eta_{i}(g)=\# N_{i}(g)$ is called the degree of player $i$.

Players have preferences over networks. With the usual assumptions on preferences, the profile of utility functions is denoted by $u(g)=\left(u_{1}(g), u_{2}(g), \ldots, u_{n}(g)\right)$, where $u_{i}$ is a mapping from $G$ to $\mathbb{R}$ for all $i \in N$. The decision of adding or deleting links is based on the marginal utility of each link. We denote the marginal utility of deleting a set of links $l \subseteq g$ from $g$ as $\Delta u_{i}(g, l):=u_{i}(g)-u_{i}(g-l)$, and similarly the marginal utility of adding a set of links $l \subseteq g^{N}-g$ to $g$ as $\Delta u_{i}(g+l, l)=u_{i}(g+l)-u_{i}(g)$. Observe that in this definition, $u_{i}(g)$ may include any kind of disutilities arising in network $g$ such as costs of link formation. In many examples from the literature linear costs of link formation are assumed, such that the utility function has the form $u_{i}(g)=v(g)-c \eta_{i}(g)$, where $c>0$ is some constant. 
Altogether, we will call $\mathbb{G}=(N, G, u)$ a society.

\subsection{Network Formation and Stability}

The study of equilibrium/stability of networks has been a subject of interest in many models of network formation. Depending on the rules of network formation which are assumed in a given model, there are many definitions of equilibrium at hand. Here, we present only the well-known concept of pairwise stability introduced by Jackson and Wolinsky $(1996){ }^{2}$

\section{Definition 1 (Pairwise Stability):}

A network $g$ in a society $\mathbb{G}=(N, G, u)$ is pairwise stable (PS) if

(i) $\forall i j \in g: \Delta u_{i}(g, i j) \geq 0$ and $\Delta u_{j}(g, i j) \geq 0$;

(ii) $\forall i j \notin g: \Delta u_{i}(g+i j, i j)>0 \Rightarrow \Delta u_{j}(g+i j, i j)<0$.

This approach to stability defines desired properties directly on the set of networks. The implicit assumption of network formation underlying this approach is that players are in control of their links; any player can unilaterally delete a given link, but to form a link both involved players need to agree. The networks which satisfy property (i) of Definition 1 are called link deletion proof and the networks which satisfy (ii) are called link addition proof.

The intuition behind the definition of pairwise stability is that two players form a link if one is strictly better off and the other is not worse off when forming the link, while a link is deleted if one of the two involved players is better off deleting the link. It should be noted that this definition of stability is rather a necessary condition of stability as it is fairly weak. It can be refined to account for multiple link deletion, called Pairwise Nash stability (Bloch and Jackson, 2006), to account for network formation with transfers, called Pairwise stability with transfers (Bloch and Jackson, 2007), and many more (see e.g. Jackson, 2008; Hellmann and Staudigl, 2014, for a further discussion on different approaches to stability). ${ }^{3}$

\footnotetext{
${ }^{2} \mathrm{~A}$ game theoretic foundation and a comparison of the several definitions of stability can be found in Bloch and Jackson (2006).

${ }^{3}$ Some results presented here generalize to the stronger concept of pairwise Nash stability, also know as pairwise equilibria. Pairwise Nash stable networks are immune against deletion of any subsets of own links.
} 


\subsection{Homogeneity}

The central assumption underlying this paper is homogeneity of the society. That is we assume all players to be ex-ante equal in order to assure that differences in utility of two players in a given network solely depend on their respective network positions but not on their name. ${ }^{4}$ We will establish this homogeneity via an anonymity condition on the utility profile.

\section{Definition 2 (Anonymity):}

Let $g_{\pi}:=\{\pi(i) \pi(j) \mid i j \in g\}$ be the network obtained from a network $g$ by some permutation of players $\pi: N \rightarrow N$. A profile of utility functions is anonymous if

$$
u_{i}(g)=u_{\pi(i)}\left(g_{\pi}\right)
$$

A society $\mathbb{G}$ with a profile of utility functions satisfying anonymity will be called homogeneous. As noted above, players in a homogeneous society are anonymous in the sense that players in symmtric network positions receive the same utility. The notion of symmetric position in a network, implied by Definition 2, is such that two players $i, j \in N, i \neq j$ are symmetric in a network $g \in G$ if there exists a permutation of the set of players $\pi: N \rightarrow N$ such that $\pi(i)=j$ and $g_{\pi}=g$. This is most trivially satisfied if two players $i, j \in N, i \neq j$ share the same neighbors (disregarding a possible common link), i.e. $N_{i}\left(g_{-j}\right)=N_{j}\left(g_{-i}\right)$. On the other hand, having the same degree is a necessary condition for two players to be in a symmetric position.

Consequently, a network $g \in G$ is called a symmetric network if all players are in a symmetric position. ${ }^{5}$ Hence, a necessary condition for $g$ to be symmetric is that it is regular, i.e. that all players have the same degree. However, this condition is not sufficient (see Figure 1). Some examples of symmetric positions in a network and symmetric networks are given in Figure 1.

Moreover, with the notion of homogeneous society, it is easy to see that symmetric links provide the same marginal utility. For this, however, a symmetry on links has to be imposed. To simplify things, note that for two players whose neighborhood coincides (disregarding a mutual connection), any link to a third player is symmetric which implies (ii) and (iii) of Lemma 1.

\footnotetext{
${ }^{4}$ In the setup at hand, ex-ante means before any network is formed.

${ }^{5}$ The graph theoretic equivalent to symmetric graphs we consider here are not symmetric, but vertex-transitive graphs. In this setup, we need symmetry of the players, that is symmetry of vertices whereas symmetry in graph theory would also demand edges to be symmetric. For details see e.g. Biggs (1994).
} 


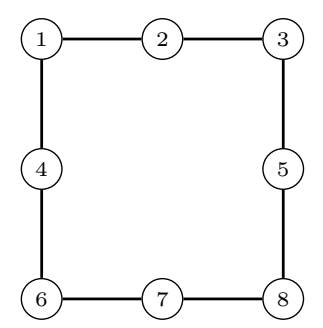

(a) Symmetric network

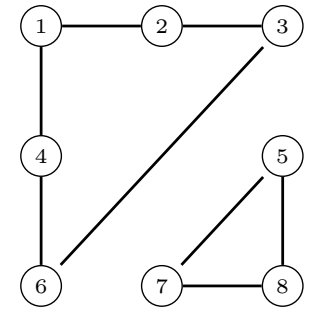

(b) Non-symmetric network

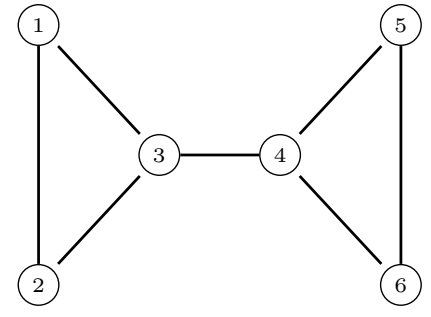

(c) Non-symmetric network

Figure 1: Networks (a) and (b) are regular, but only (a) is symmetric. In network (b), two players of different components are not in symmetric positions. In network (c), players 3 and 4 respectively players 1,2,5 and 6 are symmetric, while the network is obviously not.

\section{Lemma 1.}

Let some profile of utility functions u satisfy anonymity. Then the following statements are true:

(i) $u_{i}(g)=u_{j}(g)$, if $i$ and $j$ are symmetric,

(ii) $\Delta u_{i}(g+i k, i k)=\Delta u_{j}(g+j k, j k) \quad \forall k \in N \backslash N_{i}(g)$, if $N_{i}\left(g_{-j}\right)=N_{j}\left(g_{-i}\right)$,

(iii) $\Delta u_{k}(g+i k, i k)=\Delta u_{k}(g+j k, j k) \quad \forall k \in N \backslash N_{i}(g)$, if $N_{i}\left(g_{-j}\right)=N_{j}\left(g_{-i}\right)$.

The proof of Lemma 1 as well as all following results can be found in the appendix. From the proof it can be easily seen that parts (ii) and (iii) of Lemma 1 hold likewise for all existing links $i k, j k \in g$.

\subsection{Link externalities}

Even if the society is homogeneous, pairwise stable networks may fail to exist. Moreover, it is impossible to say anything about stability of particular network structures without any assumptions on the utility function. In the literature on network formation, however, many utility functions admit certain link externality conditions. By link externalities we mean conditions on how marginal utility is affected when links are added to or deleted from a network. Hence, without losing much of the generality of our approach, we will examine whether stable networks of certain structure exist if various combinations of link externalities in the context of homogeneous societies are satisfied. We will consider the weakest version of link externalities in the literature, namely the ordinal versions presented in Hellmann (2013). ${ }^{6}$ For the sake of convenience, in the rest of the paper we will

\footnotetext{
${ }^{6}$ Ordinal link externalities as first defined by Hellmann (2013) are implied by the more com-
} monly used but stronger cardinal link externalities (see e.g. Bloch and Jackson, 2006, 2007; 
speak about convexity, concavity, strategic complements and strategic substitutes, keeping in mind that what is used are the respective ordinal formulations of Definition 3.

\section{Definition 3 (Ordinal link externalities):}

A utility function $u_{i}$ satisfies ordinal convexity (concavity) in own links if for all $g \in G, l_{i} \subseteq L_{i}\left(g^{N}-g\right)$ and $i j \notin g+l_{i}$ it holds that

$$
\Delta u_{i}(g+i j, i j) \geq 0 \Rightarrow(\Leftarrow) \Delta u_{i}\left(g+l_{i}+i j, i j\right) \geq 0 .
$$

A utility function $u_{i}$ satisfies ordinal strategic complements (substitutes) if for all $g \in G, l_{-i} \subseteq L_{-i}\left(g^{N}-g\right)$ and $i j \in L_{i}\left(g^{N}-g\right)$ it holds that

$$
\Delta u_{i}(g+i j, i j) \geq 0 \Rightarrow(\Leftarrow) \Delta u_{i}\left(g+l_{-i}+i j, i j\right) \geq 0 .
$$

In Goyal and Joshi (2006b) two utility functions with a particular structure called playing the field and local spillovers- are studied with respect to existence of stable networks. Both of these utility functions reduce the network to only one characteristic: the vector of degrees, which reduces complexity a lot, but takes away the generality and hence a whole field of possible applications. To establish existence of stable networks, Goyal and Joshi (2006b) additionally assume various combinations of cardinal notions of link externalities. It is straightforward to see that our assumptions of homogeneity and ordinal link externalities are implied by theirs. Hence not only with respect to not assuming a particular structure, but also with respect to the notions of link externalities, our approach is a true generalization of their approach and offers new opportunities to apply the results.

\subsection{Example: Utility given by Bonacich Centrality}

We illustrate our assumptions and results with the help of an example where players have a desire to be central in a network. This also reflects the first ideas of why individuals form links strategically (see e.g. Jackson and Wolinsky, 1996). What exactly is meant by being central very much depends on the definition of centrality (for a discussion and comparison of different measures of centrality, see e.g. Jackson, 2008). In Jackson and Wolinsky's influential connections models players derive utility based on a version of decay or closeness centrality.

Network theory offers a wide variety of centrality measures, and some of them have an interesting game theoretic interpretation. Bonacich (1987) introduced a

Goyal and Joshi, 2006b), as well as by several related concepts such as $\alpha$-submodularity

(Calvó-Armengol and Ilkiliç, 2009). 
parametric family of centrality measures in order to formulate the intuitive idea that the centrality of a single node in a network should depend on the centrality of its neighbors. This self-referential definition of centrality leads to an eigenvectorbased measure, which can be derived from basic utility-maximization ideas, as shown by Ballester et al. (2006). Let $A$ be the $n \times n$ adjacency matrix of a given network $g$ and $\overrightarrow{1}$ be the $n \times 1$ vector with all entries equal to $1 .^{7}$ The powers of the adjacency matrix yield information about the connectivity structure of the network. Indeed, $A \overrightarrow{1}$ is an $n \times 1$ matrix whose entries are just the degrees of the individual nodes. The vector $A^{2} \overrightarrow{1}$ counts the number of paths of length 2 starting from the individual nodes, and more generally $A^{k} \overrightarrow{1}$ counts the number of paths of length $k$. Let $\delta>0$ be a given parameter, discounting for path length and chosen in such a way that the following matrix power series exists: ${ }^{8}$

$$
B(\delta, g)=\sum_{n=0}^{\infty} \delta^{n} A^{n}=[I-\delta A]^{-1} .
$$

The centrality index proposed by Bonacich (1987) is then defined as

$$
b(\delta, g)=B(\delta, g) \overrightarrow{1} .
$$

This centrality measure is actually a Nash equilibrium of an interesting class of non-cooperative games: Suppose there are $N$ agents who are involved in a team production problem (for an indepth introduction of this game, see Ballester et al., 2006). Each player chooses a non-negative quantity $x_{i} \geq 0$, interpreted as efforts invested in the team production. Efforts are costly, and the level of effort invested by the other players affects the utility of player $i$. To capture these effects, let the player $i$ 's payoff from an effort profile $x=\left(x_{1}, \ldots, x_{N}\right)$ be given by

$$
\pi_{i}\left(x_{1}, \ldots, x_{N}\right)=x_{i}-\frac{1}{2} x_{i}^{2}+\delta \sum_{j \in N_{i}} x_{i} x_{j} .
$$

The players choose their efforts independently, and in a utility maximizing way. It can be shown that this game has a unique Nash equilibrium $x^{*}$ given by

$$
x^{*}=b(\delta, g) .
$$

Hence, the equilibrium effort invested by player $i$ depends only on her centrality in the network. Given the network $g$, and discount factor $\delta \in \mathbb{R}$, so that (2.4) is well defined, the equilibrium payoff of player $i$ can be computed as ${ }^{9}$

$$
\pi_{i}\left(x^{*}\right)=\frac{1}{2} b_{i}(\delta, g)^{2} .
$$

\footnotetext{
${ }^{7}$ The adjacency matrix $A$ of a network $g$ is a matrix with entries $a_{i j}=1$ if $i j \in g$ and $a_{i j}=0$ otherwise. Note that $A$ is necessarily symmetric as we consider undirected network formation.

${ }^{8}$ The necessary condition for this to be the case is that $0<\delta<\lambda_{1}(A)^{-1}$, where $\lambda_{1}(A)$ is the eigenvalue of $A$ having largest modulus.

${ }^{9}$ To see this, note that $b_{i}(g, \delta)=1+\delta \sum_{j \in N_{i}} b_{j}(g, \delta)$.
} 
This utility function now represents preferences over a set of possible network architectures underlying the team production problem. Hence, assuming Nash equilibrium play in the game where players choose efforts, we can now use this derived preference relation to investigate the equilibrium payoffs as functions of the interaction structure. In fact, we can find many examples in the literature where the equilibrium outcome of a game on a network is given by the Bonacich centrality. Among those are models of production economy (Acemoglu et al., 2012), R\&D cooperation (König, 2012), local public goods (Allouch, 2012; Bramoullé et al., 2014), and trade (Bosker and Westbrock, 2014).

Thus, in a stage game such that players first decide to form costly links and then choose efforts in a team production game, players will anticipate the equilibrium in the second stage. Hence when they form links with linear costs the following objective function arises,

$$
u_{i}^{B C}(g)=\frac{1}{2} b_{i}(\delta, g)^{2}-\eta_{i} c
$$

When considering link formation with the utility function $u_{i}^{B C}(g)$ as the objective, we have to make sure that $b_{i}(g, \delta)$ is well defined for any network. Since the largest eigenvalue $\lambda_{1}(g)$ is maximized for the complete network $g^{N}$, and we need $\delta<\frac{1}{\lambda_{1}(g)}$ for $b_{i}(g, \delta)$ to exist, we have to assume

$$
\delta<\frac{1}{\lambda_{1}\left(g^{N}\right)}=\frac{1}{n-1}
$$

in order to define a consistent model of network formation. In other words, the set of admissible discount factors is given by $\delta \in\left[0, \frac{1}{n-1}\right)$.

This profile of utility functions obviously satisfies anonymity. Moreover, it is quite intuitive to see that the Bonacich centrality $b_{i}(g, \delta)$ satisfies positive link externalities, i.e. convexity and strategic complements as in Definition 3, since more own or other players' links increase the number of paths that a new link creates. Since a convex transformation does not change this fact and linking costs are linear, marginal utility is increasing in own and other players' links.

It is worth noting that to our best knowledge, there is only one result from the literature that can be applied to shed some light into the structure of pairwise stable networks when individuals form links according to $u_{i}^{B C}$. From Hellmann (2013) it is known that a pairwise stable network exists. Other models are not applicable, since $u_{i}^{B C}$ does not fall in the category of playing the field and local spillover games of Goyal and Joshi (2006b), and does not allow for a network potential (cf. Jackson and Watts, 2001; Chakrabarti and Gilles, 2007). Hence, with our general assumptions of this paper, we are able to offer some insights into the structure of pairwise stable networks of this type of utility function. 


\section{Strategic Complements}

In this section we assume that the profile of utility functions satisfies the ordinal notion of strategic complements. Such link externalities are given if the incentives to form links are single crossing in other players' links in the sense that once the incentive to form a link is positive, it stays positive when links of other players are added. The more restrictive cardinal notion of strategic complements would imply that the incentive to form links is increasing in other players' links. Hence there is a form of complementarity between links at work: Links to other players become more valuable when links between other players are added.

However, there are two kinds of link externalities which are not captured by the assumption of ordinal strategic complements. First, it is not clear what the effect of own links is on incentives to form links. When these are negative, this could potentially lead to cycling behavior. ${ }^{10}$ Second (and this cannot be captured by the cardinal notion either), strategic complements do not specify on which links the effect of other players' links is stronger. That is, if two players $k$ and $l$ form a link, does this increase the incentive for player $i \notin\{k, l\}$ more to link to $k$ (resp. $l)$ than to $j \notin\{k, l\}$, or vice versa?

To capture these different externalities in a homogeneous society, we will first assume that additionally to strategic complements, incentives from own links are not "too negative" in a well defined sense (Definition 4). With these assumptions an already strong existence result can be established which trivially also holds for the case when both link externalities are positive. We then show that in such an environment, it is possible to characterize a class of networks to which all pairwise stable networks belong, if the society is more homogeneous. By that, we mean that the strategic complements property and the convexity property act homogeneously on all links. In the case of strategic complements, this results in the fact that players prefer to connect to players with higher degree. We call this a strong preference for centrality (see Section 3.2) since this reflects a preference to be central in the network. These assumption is not far-fetched. We discuss examples satisfying it, among them the utility function where benefits are given by Bonacich centrality, i.e. $u_{i}^{B C}$. Finally, we show in Section 3.3, that in a homogeneous society, strategic complements alone (in settings where the utility functions depends on the vector of degrees and the network structure) are sufficient for the existence of a pairwise stable network within the class of symmetric networks.

\footnotetext{
${ }^{10}$ In the opposite case of both externalities from own and other players links being positive, it is shown in Hellmann (2013) that closed cycles do not exist (see Jackson and Wolinsky, 1996, for a definition of closed improving cycles).
} 


\subsection{Link Monotonicity}

When the incentives to form links are increasing in both own and other players' links, then network formation is reminiscent of the structure of a supermodular game, where equilibria are easy to characterize. However, pairwise stable networks are not necessarily Nash equilibria of an underlying game. ${ }^{11}$ We show here that the idea of assuming increasing incentives, i.e. positive link externalities, can be relaxed in two ways: first, strategic complements only need to hold in ordinal terms, and second, externalities from own links may not satisfy the single crossing property, but instead shall not be "too negative". In particular, we want the potential negative effect of adding own links not to dominate the positive effect of addition of other players' links. This idea is inspired by the notion of link monotonicity in Goyal and Joshi (2006b). Their notion can be generalized to our general utility function and to only hold in ordinal terms. We formally define that a utility profile satisfies ordinal link monotonicity if the addition of an own link and some other player's link to any given network at the same time does not turn marginal utility negative for any player.

\section{Definition 4 (Link Monotocity):}

A utility function $u_{i}$ satisfies ordinal link monotonicity if for all $j, k, l, m \in N \backslash\{i\}$ and all $g \in G$ :

$$
\Delta u_{i}(g+i j, i j)>(\geq) 0 \Rightarrow \Delta u_{i}(g+i k+l m+i j, i j)>(\geq) 0,
$$

Trivially, if externalities from own and other players' links are both positive (cf. Definition 3), then ordinal link monotonicity is satisfied, but not vice versa. Also our notion of ordinal link monotonicity is implied by the assumption of link monotonicity in Goyal and Joshi (2006b).

Now, in a homogeneous society, if the empty network is not stable, then any two players want to connect to each other (cf. Lemma 2). In the presence of link monotonicity and strategic complements, this implies that a player with less own links than the total number of other players' links, has an incentive to add any link. We then show that if the number of players $n$ is at least five, then there always exist two unconnected players satisfying the above, what implies that they both want to connect to each other. Hence, only the complete network can be stable which is summarized in the following result.

\footnotetext{
${ }^{11}$ The non-cooperative game underlying network formation is due to Myerson (1991), where the intentions to form links are announced. Nash equilibria of this game are immune to multiple link deletion and do not consider link addition.
} 


\section{Proposition 1.}

Let $n>4$ and let the profile of utility functions $u$ satisfy the ordinal strategic complements property, ordinal link monotonicity and anonymity. If the empty network is not pairwise stable then the complete network is uniquely pairwise stable, and vice versa.

Hence, if the society is homogeneous and ordinal strategic complements dominate externalities from own links such that link monotonicity is satisfied, then the pairwise stable networks have an interesting structure: if multiple networks are pairwise stable, then there always exists a smallest and a largest stable network in the sense of the set inclusion ordering, namely the empty and the complete network. To the contrary, if one of these networks fails to be pairwise stable, then the other network is uniquely pairwise stable, i.e. the least and maximal network coincide.

Note that the assumptions in Proposition 1 allow for negative effects from both own and other players' links and that even concave utility functions are allowed as long as the ordinal properties of strategic complements and link monotonicity are preserved. Hence, the range of possible applications is large, establishing a strong existence result. In such network formation models, it suffices to check the empty and the complete network in order to find a pairwise stable network. Especially in large societies, where the number of different networks is enormous ${ }^{12}$ this offers an easy way to find a stable network.

As a direct consequence of Proposition 1 we get the same result in case of ordinal positive externalities since convexity and strategic complements imply ordinal link monotonicity. In this case the result also holds for $n \leq 4$.

\section{Corollary 1.}

Let the profile of utility functions $u$ satisfy the ordinal strategic complements property, ordinal convexity in own links and anonymity. If the empty network is not pairwise stable then the complete network is uniquely pairwise stable, and vice versa.

A comparison to the literature may be in order here. First, Goyal and Joshi (2006b) assume a lot more structure on the functional form of utility and combine these with cardinal assumptions of link externalities. Although our approach is more general, we are able to contribute more concerning the stability of complete and empty network (cf. Goyal and Joshi, 2006b, Proposition 4.1). ${ }^{13}$ Second,

\footnotetext{
${ }^{12}$ In a society of $n$ agents, the cardinality of $G$ is $2^{n(n-1) / 2}$.

${ }^{13}$ Note that Goyal and Joshi (2006b) do not get the same since their focus is on existence of pairwise Nash stable networks rather than pairwise stable networks.
} 
Hellmann (2013) studies the same assumptions on link externalities as Corollary 1 , but for heterogeneous societies. There, only existence can be established, implying that the homogeneity assumption has some impact here.

As noted in Section 2.4, when benefits are given by a convex transformation of Bonacich centrality and link costs are linear (2.6), then positive link externalities and anonymity are satisfied. Hence, by Corollary 1, the empty or the complete network are uniquely stable or that both are stable in this setting.

\subsection{Centrality-based Utility Functions}

Although it is possible to gain some insights into the structure of pairwise stable networks in a homogeneous society when ordinal link externalities are not too negative, these assumptions are not sufficient to characterize all pairwise stable networks. In particular, it would be interesting to examine which stable structures emerge when the least and maximal stable network do not coincide, such that multiple stable networks exist. However, in the general framework that we impose here there is little hope to say more about the structure of pairwise stable networks without putting stronger assumptions on the utility function.

The basic idea behind network formation starting from the seminal contribution Jackson and Wolinsky (1996) is that players have a desire to be as central as possible in the network. In these settings, players prefer the connection to a central player over the connection to a peripheral player. We reflect this idea by defining centrality based utility functions by a weak notion and a strong notion.

\section{Definition 5 (Weak and Strong Preference for Centrality):}

A utility function $u_{i}$ satisfies weak preference for centrality (WPC) if for all $g \in G$, whenever there exist $j, k \in N \backslash N_{i}(g)$ such that $N_{j}\left(g_{-k}\right) \subseteq N_{k}\left(g_{-j}\right)$ it holds that

$$
\Delta u_{i}(g+i j, i j) \geq(>) 0 \quad \Rightarrow \quad \Delta u_{i}(g+i k, i k) \geq(>) 0,
$$

A utility profile $u_{i}$ satisfies strong preference for centrality (SPC) if for all $g \in G$, $\eta(g) \in\{0, . ., n-1\}^{n}$ such that $\eta_{j}(g) \leq \eta_{k}(g)$ it holds that

$$
\Delta u_{i}(g, i j) \geq 0 \Rightarrow \Delta u_{i}(g+i k, i k)>0 .
$$

Our weak notion of preference for centrality captures all reasonable notions of centrality based utility functions: player $k$ is more central than $j$ if $k$ 's neighbors are a superset of $j$ 's, and hence any player, who has an incentive to connect to $j$, also has an incentive to connect to $k$. The definition of weak preference for centrality, hence, represents a necessary condition for centrality cased utility 
functions, ${ }^{14}$ and thus leaves room for many utility functions, also for those which are not directly concerned with centrality itself. Examples of utility functions satisfying WPC are e.g. the connections model (Jackson and Wolinsky, 1996), and the utility function with Bonacich centrality as the objective (cf. Section 2.4).

The notion of strong preference for centrality is more demanding: player $i$ has an incentive to connect to $k$ if $i$ has an incentive to connect to $j$ and $k$ has more neighbors than $j$. Expressed in cardinal terms, this means that a player prefers to connect players with higher number of neighbors. ${ }^{15}$ To interpret this definition in terms of link externalities, consider a stronger notion of homogeneity such that players have the same incentive to connect to players with the same degree. If this is the case then it is easy to see that SPC is implied by ordinal strategic complements. Hence, the condition of SPC can be satisfied in terms of more homogeneous societies when utility satisfies strategic complements.

To capture externalities from own links consider the following notion of anonymous convextiy.

\section{Definition 6 (Anonymous Convexity):}

A utility profile $u$ satisfies anonymous convexity (AC) if for all $g \in G, \eta(g) \in$ $\{0, . ., n-1\}^{n}$ such that $\eta_{i}(g) \leq \eta_{j}(g)$, it holds that

$$
\Delta u_{i}(g, i k) \geq 0 \Rightarrow \Delta u_{j}(g+j k, j k) \geq 0 .
$$

Anonymous convexity is a somehow stronger notion of ordinal convexity since it implicitly assumes a higher degree of homogeneity (similarly to above): if a player $i$ likes the connection to $k$ then any player with more links also has an incentive to connect to $k$. In a more homogeneous society where players with same degree have the same incentives, this formulation reflects the idea of ordinal convexity since once the marginal utility of a link is positive, it stays positive if own links are added. Hence anonymous convexity translates the convexity notion to other players.

Recall that we aim at characterizing a class of networks which incorporates all pairwise stable networks. The set of networks that we will need is given by the following definition.

\footnotetext{
${ }^{14}$ We mean here necessary conditions for centrality based utility functions in terms of connectivity. To the contrary, utility functions based on betweenness centrality where players have an incentive to locate at structural holes may not satisfy weak preference for centrality, see also Goyal and Vega-Redondo (2007).

${ }^{15}$ Note further that in the definition of SPC, we used the fact that $i j$ is already in $g$ such that after the addition of the link $i k$ player $k$ has indeed strictly more links. Defining SPC (Definition 3.3) this way helps simplifying notation since we do not have to deal with distinguishing weak and strict inequalities for several cases. AC (Definition 6) is analogously defined.
} 


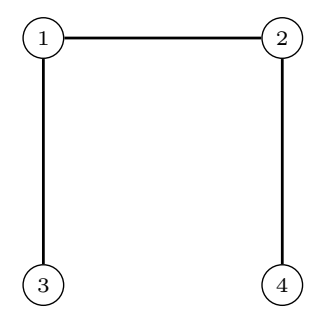

(a) $P_{4}:$ Path of length 4

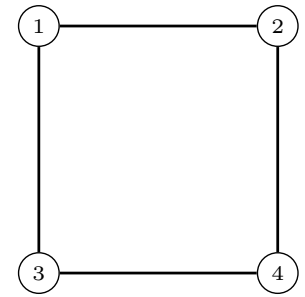

(b) $C_{4}: \quad$ Cycle of size 4
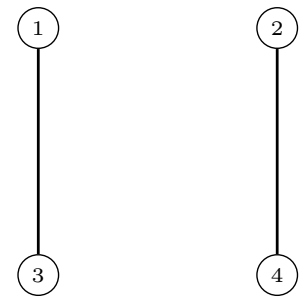

(c) $K_{2,2}$ : Two complete size 2 components

Figure 2: A network is a nested split graph if it does not contain a set of four players who form one of the subgraphs $P_{4}, C_{4}, K_{2,2}$.

\section{Definition 7 (Nested Split Graphs):}

A network $g \in G$ is a nested split graph (NSG) if for all players $i, j, k \in N$ such that

$$
\eta_{i}(g) \geq \eta_{j}(g) \geq \eta_{k}(g)
$$

we have that if $i k \in g$ then also $i j \in g$ and if $j k \in g$ then also $i k \in g$.

In a nested split graph the neighborhood structure of all players is nested in the sense that for any two players $i, j \in N$ the set of their neighbors can be ordered according to the set inclusion order, i.e. $N_{i}\left(g_{-j}\right) \subseteq N_{j}\left(g_{-i}\right)$ or $N_{i}\left(g_{-j}\right) \supseteq N_{j}\left(g_{-i}\right)$. Our Definition 7 can be straightforwardly seen to be equivalent to the ones in Cvetković and Rowlinson (1990), Mahadev and Peled (1995), and Simić et al. (2006). In particular, a network is NSG if and only if it does not contain a path $\left(P_{4}\right)$, a cycle $\left(C_{4}\right)$ or two connected pairs $\left(K_{2,2}\right)$ when restricted to any 4 players (see Figure 2). Moreover, nested split graphs maximize the largest eigenvalue of networks that contain the same number of links. ${ }^{16}$

More importantly for our purposes, the set of nested split graphs contains all pairwise stable networks when the profile of utility functions satisfies SPC and AC.

\section{Proposition 2.}

Suppose a profile of utility functions satisfies strong preference for centrality and anonymous convexity. Then any pairwise stable network is a nested split graph.

Although the utility function is not specified in our framework, we learn a lot about the structure of pairwise stable networks: in a pairwise stable network we can order any two players' neighbors with respect to the set inclusion order when

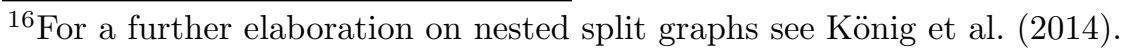


SPC and AC are satisfied. This reduces the set of possible candidates for PS networks considerably as the set of NSG's only make up a very small fraction of the set of all possible networks $G$.

The assumptions needed in this result may seem demanding at first sight. However, the conditions of SPC and AC may very naturally be implied by the other notions of link externalities. To see this most easily, consider again the framework of Goyal and Joshi (2006b). There, both conditions SPC and AC are automatically satisfied in both playing the field and local spillover games, when assuming convexity and strategic complements. Hence, in more homogeneous societies, these notions are implied by positive link externalities. In particular, the example of provision of a pure public good in Goyal and Joshi (2006b), inspired by a model of Bloch (1997), satisfies the assumptions of Proposition 2. Note also that by SPC and AC we just assumed ordinal notions, such that negative effects from adding links can still occur, as long as the single crossing properties of these definitions are preserved.

Further, with our general approach we are able to study interesting utility functions which do not fall into the class of playing the field or local spillover games in Goyal and Joshi (2006b). One such example is given by the important class of utility where players strive for maximizing their Bonacich centrality given by (2.6). In fact, it is possible to show that for low enough discount factors the utility profile $u^{B C}$ satisfies SPC and AC and therefore pairwise stable networks are of nested split architecture.

\section{Corollary 2.}

The profile of utility functions $u^{B C}$ defined by (2.6) satisfies strong preference for centrality and anonymous convexity for any discount factor $\delta<\frac{1}{(n-1)^{2}}$.

Although the utility function given by the Bonacich centrality seems to be quite a complex object since it considers the infinite discounted sum of all possible paths in the networks, it is possible to characterize the set of pairwise stable networks at least for low enough discount factors. This is due to the fact that $u^{B C}$ satisfies SPC for these low discount factors since the benefits from second order connections (degree of neighbors) dominate any benefits from higher order connections which is shown in the proof of Corollary 2. Hence, although our results hold for general utility functions, they are still applicable to interesting classes of utility functions and help characterize the structure of PS networks, even where no results are available so far. 


\subsection{Existence of Symmetric Networks}

A natural question that may arise when studying homogeneous societies is whether we always get existence of symmetric network structures which are pairwise stable, since in symmetric networks all players receive the same utility by Lemma 1 . However, incentives to form different links may differ even in symmetric networks, since in our notion a symmetric network is vertex transitive but not edge transitive (the former always exists for any degree, see Lemma 2, while especially for high degrees there may not exist edge transitive networks). In the previous section, we did get existence of symmetric networks since either the empty or the complete network is always pairwise stable, although the structure of stable networks in general can be quite asymmetric (see e.g. Proposition 2).

In this section, we will show that strategic complements alone is sufficient to establish the existence of a pairwise stable network of symmetric architecture for a broad range of utility profiles. To establish the existence result we require that a symmetric network of any degree exists. Since existence of regular networks for all possible degrees only holds if the number of nodes $n$ is even and regularity is necessary for symmetry, we will first assume an even number of players. If $n$ is even, then it is indeed possible to show that according to our definition of symmetry (i.e. vertex-transitive graphs, cf. Section 2.2), there also exist symmetric networks of any degree.

Lemma 2 (Existence of symmetric networks).

Suppose the number of players $n=|N|$ is even. Then for any degree $p$ such that $0 \leq p \leq n-1$, there exists a symmetric network $g_{p}^{r}$. Hence, for anonymous utility functions there exists for any degree $p$ a network $g_{p}^{r}$ which satisfies that $u_{i}\left(g_{p}^{r}\right)=u_{j}\left(g_{p}^{r}\right)$ for all $i, j \in N$.

In the proof we construct a sequence of symmetric bipartite networks starting from the empty network until the complete symmetric bipartite network (of degree $\frac{n}{2}$ ) is reached, from which the respective complements are again symmetric and reach the complete network. Notice that this construction does not represent a sequence of link addition leading from the empty to the complete network. There is a rearrangement of links when moving from the complete bipartite network $g_{n / 2}^{b i p}$ to the complement of the bipartite network $g_{n / 2-1}^{b i p}$, as illustrated in Figure 3. In general, it is straightforward to see that a sequence of link addition encompassing symmetric networks of every degree does not exist. ${ }^{17}$

In the proof of the following result we make use of such link addition paths to the

${ }^{17}$ Note to the contrary, we can always construct a link addition sequence encompassing regular networks of all degrees. 


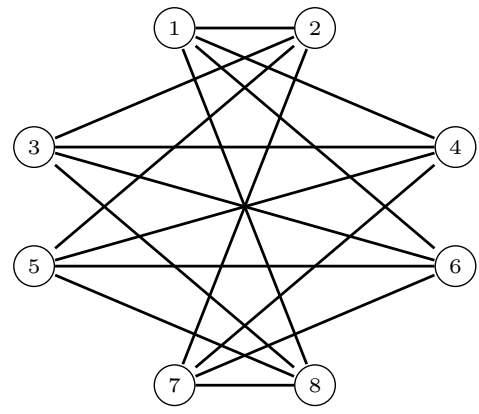

(a) $g_{n / 2}^{b i p}$

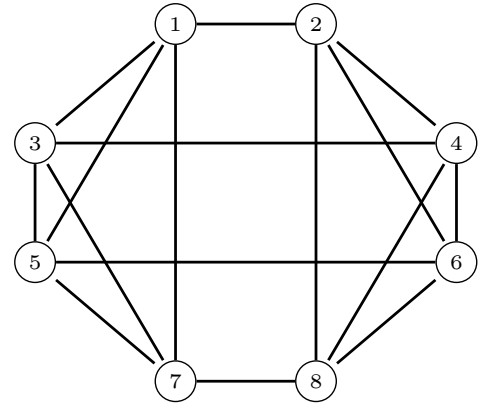

(b) $g^{N}-g_{3}^{b i p}$

Figure 3: The complete symmetric bipartite network $g_{n / 2}^{b i p}$ and a network of same degree for which the complement is bipartite.

point where the complete bipartite network $g_{n / 2}^{b i p}$ is reached to apply the strategic complements property. Thus we need to make an additional assumption to assure that on the one hand it is possible to choose the path that leads to the symmetric network of next higher degree. On the other hand, we need to ensure that when switching from the complete bipartite network to a complement of a symmetric bipartite network of same degree then deletion the incentive to keep links do not turn negative. A straightforward (and certainly not the most general) way to guarantee this is to assume degree-based utility profiles, such that utility of any player has the form

$$
u_{i}(g) \equiv u_{i}\left(\eta_{i}(g),\left(\eta_{j}(g)\right)_{j \in N_{i}(g)},\left(\eta_{k}(g)\right)_{k \notin N_{i}(g)}\right) .
$$

While this assumption seems demanding as utility now solely depends on the degree of players, the great majority of examples in the literature complies with it, including all utility profiles in Goyal and Joshi (2006b).

\section{Proposition 3.}

Suppose the number of players $n$ is even and the profile of degree-based utility functions satisfies strategic complements and anonymity. Then there exists a symmetric network which is pairwise stable.

The assumption of the utility profile being degree-based can be interpreted as a strengthening of the anonymity assumption. In case of an anonymous utility profile players do not discriminate between others that are in symmetric network positions and where both links are edge symmetric. Here, players discriminate neither between players of same degree they are connected to, nor between players of same degree they are not connected to.

Hence, we generically arrive at a general result: there always exists a symmetric network if strategic complements are satisfied. The driving force of existence of 
a symmetric network seems to be the anonymity assumption alongside with the condition of strategic complements. In settings where strategic complements are not satisfied, it is easy to show that there might not exist a stable symmetric network in a homogeneous society (this even holds in the reduced framework of Goyal and Joshi (2006b), see also Section 4).

For Proposition 3 it is necessary to assume that the number of players $n$ is even. Otherwise there do not exist symmetric networks for every degree. In the appendix, we show that for societies of an odd number of players that almost symmetric networks are stable if we additionally assume weak preference for centrality.

Because of the construction of link addition in Proposition 3, we can deduce as a corollary of Proposition 3 the existence of a symmetric stable bipartite network in a framework of two-sided network formation. Suppose there are two groups (e.g. buyers and sellers) of the same size. Links can only be formed across both groups such that the set of all networks is restricted to the set of bipartite networks, $G_{n / 2}^{b i p}:=\left\{g \mid g \subseteq g_{n / 2}^{b i p}\right\}$. Such a network formation model of buyers and sellers is formally introduced in Kranton and Minehart (2001), see also Polanski and Vega-Redondo (2013). Another example are two-sided matching markets, see e.g. to Roth and Sotomayor (1992). When network formation is restricted to links across two groups, it is trivially possible to apply the insights of Lemma 2 and Proposition 3. Hence, existence of a stable symmetric bipartite (buyer seller) network is guaranteed, and we get the following result.

\section{Corollary 3.}

Consider network formation of a homogeneous society $\mathbb{G}:=\left(N, G_{n / 2}^{b i p}, u\right)$ where the profile of utility functions is degree based and satisfies strategic complements. Then there always exists a symmetric network that is pairwise stable.

The proof follows directly from Proposition 3.

\section{Convexity}

We finally want to assess which networks are likely to form in homogeneous societies when strategic complements are not necessarily satisfied, but instead we assume that the utility function is convex in own links. Recall that ordinal convexity as given in Definition 3 orders the externalities of own links on marginal utility in a way that, once positive, it will stay positive whenever own links are added to the network. In presence of this form of complementarity between own links the intuition is that players that already have links are likely to strive for 
more. Notice however that due to ambiguous marginal effects of other links still cycling behavior may arise in link formation such that no pairwise stable network would exist.

To the contrary, we show in the following that with the additional assumption of WPC as in Definition 5 stable networks still exist. We find existence of stable networks in the class of dominant group networks. A network is of dominant group architecture if a subset of $0 \leq m \leq n-1$ players constitutes a completely connected subgraph, while all other $n-m$ players remain isolated. We will denote a dominant group network with a complete subgraph of size $m$ by $g_{m}^{d g}$.

\section{Proposition 4.}

Suppose the profile of utility functions satisfies convexity, anonymity and WPC as in Definition 5. Then there exists a pairwise stable network of dominant group architecture $g_{m}^{d g}$, for some $0 \leq m \leq n-1$.

The intuition for Proposition 4 is as follows. First, as marginal utility satisfies convexity, players incentive to form a link is not destroyed by additional own links. Second, they tend to connect to players that already have more links, due to WPC. Both effects together point to networks where players either have a lot or no links. In the proposition we then naturally find the stable networks in the extreme case, namely one completely connected subgroup and one subgroup of isolated players (one of these sets can be empty).

Let us emphasize again that WPC is a very weak assumption since it rather defines necessary conditions of preferences to be central in the network. Further, both WPC and convexity only need to be satisfied in ordinal terms such that the overall effect can be negative. There are many examples from the literature which satisfy Proposition 4. We take a closer look at two of those examples where stronger convexity assumptions result in dominant group networks being even the unique pairwise stable network architecture.

Example 1 (Cost-reducing collaboration in oligopoly). One classical example in the literature considers a Cournot oligopoly of $n$ firms that are supposed to be ex-ante identical, but can form bilateral collaboration links lowering their respective marginal costs (Goyal and Joshi, 2003, 2006b; Dawid and Hellmann, 2014).

The authors show that the equilibrium quantities of each firm are

$$
q_{i}(g)=\frac{\left(a-\gamma_{0}\right)+(n-1) \gamma \eta_{i}(g)-\gamma \sum_{j \neq i} \eta_{j}\left(g_{-i}\right)}{n+1}, \quad i \in N,
$$

while Cournot profits are given by $\pi_{i}(g)=q_{i}^{2}(g)$. This results in marginal profit 
of an additional link $i j \notin g$ being

$\Delta \pi_{i}(g+i j, i j)=\frac{\gamma(n-1)}{(n+1)^{2}}\left[2\left(\alpha-\gamma_{0}\right)+\gamma(n-1)+2 \gamma n \eta_{i}(g)-2 \gamma \sum_{j \neq i} \eta_{j}(g)\right]-f$,

where $f$ are the costs of forming a link. From marginal utility it can be derived that ordinal convexity is satisfied (see also Dawid and Hellmann, 2014). What is more, WPC is satisfied as firms do not discriminate between different partners when deciding with whom to link in this game. Thus we are indeed in the situation of Proposition 4 and there exists a network of dominant group architecture in this setup.

As firms however do not discriminate their neighbors by their network position and utility is (in fact) strictly convex, marginal utility even yields the following special property:

$$
\Delta u_{i}(g, i j) \geq 0 \quad \Rightarrow \quad \Delta u_{i}(g+i k, i k)>0 \quad \forall k \in N \backslash\{i\}
$$

Here, Property 4.1 is stronger than the condition of Anonymous Convexity (Definition 6). If such a strong property is satisfied in a network formation game, then it is straightforward to see that only networks of dominant group architecture can be pairwise stable.

\section{Proposition 5.}

Suppose a profile of utility functions satisfies the property given in (4.1). Then any pairwise stable network is of dominant group architecture.

The proof is rather trivial (and hence skipped in the appendix): If a network is stable, then no link can be deleted, which means that for any link $i j \in g$ we have $\Delta u_{i}(g, i j) \geq 0$ which implies that any player who has a link wants a link to any other player by (4.1). Hence, the only possible pairwise stable network architecture in this case is the dominant group architecture.

Thus, Proposition 5 implies the result by Goyal and Joshi (2003) that all pairwise stable collaboration networks are dominant group networks in the case of Cournot competition.

Example 2 (Bonacich Centrality revisited). In Section 3.2 we have shown that Bonacich utility $u^{B C}$ satisfies convexity and SPC for discount factors $\delta<$ $\frac{1}{(n-1)^{2}}$, so that we are in the situation of Proposition $4 .{ }^{18}$

However, we argue that an even stronger anonymity property will yield that dominant group networks are even the unique pairwise stable networks. Ob-

\footnotetext{
${ }^{18}$ Remember that WPC required in Proposition 4 follows from SPC.
} 
serve first that in Corollary 2 we have shown that $u^{B C}$ satisfies anonymous convexity, such that for any $i, j, k \in N$ and $\eta_{i}(g) \leq \eta_{j}(g)$ it is

$$
\Delta u_{i}^{B C}(g, i k) \geq 0 \Rightarrow \Delta u_{j}^{B C}(g+j k, j k) \geq 0 .
$$

Moreover, from equation (6.6) in the proof it can be directly seen that anonymous convexity in fact holds independently of the respective numbers of links of players $i$ and $j$, such that $u^{B C}$ actually satisfies the following stronger property

$$
\Delta u_{i}(g, i k) \geq 0 \quad \Rightarrow \quad \Delta u_{j}(g+j k, j k)>0 \quad \forall j \in N_{k}\left(g^{N}-g\right),
$$

such that if some player has an incentive to connect to $k$, then all other players also want to connect to $k$.

As in the previous example, this is a strong property since it is implied by convexity in very homogeneous societies satisfying independence of own links. Moreover, while Property (4.3) is not quite the same as Property (4.1) in the first example, it is again easy to understand that only dominant group networks can be pairwise stable.

\section{Proposition 6.}

Suppose a profile of utility functions satisfies the property given in (4.3). Then any pairwise stable network is of dominant group architecture.

Again, this is a rather trivial statement: If some player has positive marginal utility from a link to a player $k$, then anyone in the society wants to be connected to $k$ and consequently all non-isolated players have to be mutually connected.

Finally, since $u^{B C}$ satisfies Property 4.3, we can conclude that for low discount factors, any network is of dominant group architecture. Of course this does not contradict Corollary 2, as the set of dominant group networks is a subset of the set of nested split graphs.

\section{Conclusion}

In this paper the assumption of homogeneous agents is exploited in the setting of endogenous network formation to establish the existence of pairwise stable networks in presence of various combinations of link externalities.

While homogeneity was implicitly assumed in most works on existence conditions for stable networks, the main contribution of our work is to make this assumption explicit, maintaining an otherwise very general setup. We thus have been able to 
show that the driving force for existence are indeed the respective link externality conditions.

A second contribution is the characterization of specific network architectures that emerge in presence of link externalities. In the spirit of Goyal and Joshi (2006b) the emergence of regular networks in case of strategic complements was shown, while dominant group networks are likely to emerge in case of convexity.

We even go one step further in this work. When the society becomes more and more homogeneous, not only existence is guaranteed but we are able to determine classes of networks which contain all stable networks even though we have not assumed a functional form of utility. We find many examples that benefit from such characterization since previous results are not applicable.

While the present work exhibits a focus on positive link externalities it would be interesting for future research to show similar results in case of negative link externalities. Our conjecture for the case of both concavity and strategic substitutes however is that existence of pairwise stable networks is not always guaranteed. While an example of four players not yielding a pairwise stable network is presented in Hellmann (2013), this requires heterogeneous players. In fact, it is relatively easy to show that such an example for a homogeneous society cannot be constructed for five or less players, thus this remains a task for future research.

Second, a full characterization of pairwise stable networks if utility profiles are functions of Bonacich centrality still remains an open question of highest interest. While we provide a first contribution to this goal, proving existence of a pairwise stable network for any discount factor and characterizing stable networks for low discount factors, it still remains a challenge to characterize stable networks for the rest of the set of admissible discount factors. Our conjecture is that pairwise stable networks are a subset of nested split graphs. Since Bonacich centrality is found to be the equilibrium payoff in many network formation games in the recent literature, such result would be of highest interest for the ongoing research in this area.

\section{Appendix}

Proof of Lemma 1. Let the profile of utility functions $u$ satisfy anonymity.

(i). Suppose that $i, j \in N$ are symmetric such that there exists a permutation $\pi$ with $\pi(i)=j$ and $g_{\pi}=g$. Then by anonymity, we get

$$
u_{i}(g)=u_{\pi(i)}\left(g_{\pi}\right)=u_{j}(g) .
$$

(ii). Now let $i, j \in N$ such that $N_{i}\left(g_{-j}\right)=N_{j}\left(g_{-i}\right)$. Define $\pi_{i j}$ as the permutation 
where players $i$ and $j$ switch positions, that is

$$
\pi_{i j}: N \rightarrow N, \pi_{i j}(k)=k \quad \forall k \in N \backslash\{i, j\}, \pi_{i j}(i)=j .
$$

Then since $N_{i}\left(g_{-j}\right)=N_{j}\left(g_{-i}\right)$ we have $g_{\pi_{i j}}=g$. Take now any $k \in N \backslash\{i, j\}$ and define $\tilde{g}=g+i k$. Anonymity then yields

$$
u_{i}(g+i k)=u_{i}(\tilde{g})=u_{\pi_{i j}(i)}\left(\tilde{g}_{\pi_{i j}}\right)=u_{j}(g+j k) .
$$

Then it directly follows that

$$
\Delta u_{i}(g+i k, i k)=u_{i}(g+i k)-u_{i}(g)=u_{j}(g+j k)-u_{j}(g)=\Delta u_{j}(g+j k, j k) .
$$

(iii). By the same arguments as in $(i i)$ we get

$$
u_{k}(g+i k)=u_{k}(\tilde{g})=u_{\pi_{i j}(k)}\left(\tilde{g}_{\pi_{i j}}\right)=u_{k}(g+j k) .
$$

and consequently

$$
\Delta u_{k}(g+i k, i k)=u_{k}(g+i k)-u_{k}(g)=u_{k}(g+j k)-u_{k}(g)=\Delta u_{j}(g+j k, j k) .
$$

Proof of Proposition 1. Let $g^{\emptyset}$ be not pairwise stable. Take now any $g \in G$. We need to show that $g$ is not PS unless $g=g^{N}$.

Recalling that $L_{i}(g)$ denotes the set of links in $g$ that connect $i$, we denote $l_{i}(g):=\left|L_{i}(g)\right|$ and analogously $l_{-i}(g):=\left|L_{-i}(g)\right|$.

First, consider a player $i \in N$ such that in network $g$ we have $l_{i}(g) \leq l_{-i}(g)$. By anonymity of the utility profile we have $\Delta u_{i}\left(g^{\emptyset}+i j, i j\right)>0$ for all $i, j \in N$ since the empty network is assumed to be not PS. We then get, for $\bar{L}_{-i}(g) \subseteq L_{-i}(g)$ with $\bar{l}_{-i}(g):=\left|\bar{L}_{-i}(g)\right|=l_{i}(g)$,

$$
\begin{aligned}
& \Delta u_{i}\left(g^{\emptyset}+i j, i j\right)>0 \\
\Rightarrow & \Delta u_{i}\left(L_{i}(g)+\bar{L}_{-i}(g)+i j, i j\right)>0 \\
\Rightarrow & \Delta u_{i}(g+i j, i j)>0,
\end{aligned}
$$

for all $j \in N$, where the first implication holds by link monotonicity while the latter one holds by strategic complements.

Thus, no network $g \in G$ such that there exist two players $i, j \in N$ with $i j \notin g$ and $l_{i}(g) \leq l_{-i}(g), l_{j}(g) \leq l_{-j}(g)$ can be PS since $i$ and $j$ have an incentive to connect to each other. Define the set of players that satisfy $l_{i}(g) \leq l_{-i}(g)$ by $E(g):=\left\{i \in N: l_{i}(g) \leq l_{-i}(g)\right\}$ and its complement by $E^{c}(g):=N-E(g)$. Denoting for $A \subseteq N$ by $\left.g\right|_{A}:=\{i j \in g \mid i, j \in A\}$ the network restricted to $A$, 
above reasoning implies that in order for $g$ to be PS, $\left.g\right|_{E(g)}$ must be completely connected. For the remainder of the proof assume, hence, that $\left.g\right|_{E(g)}$ is completely connected.

Now, consider $E^{c}(g)$. Note that for any network, in particular for $\left.g\right|_{E^{c}(g)}$ we have,

$$
\sum_{k \in E^{c}(g)} l_{k}\left(\left.g\right|_{E^{c}(g)}\right)=2|g|_{E^{c}(g)} \mid=2 l_{i}\left(\left.g\right|_{E^{c}(g)}\right)+2 l_{-i}\left(\left.g\right|_{E^{c}(g)}\right)
$$

for all $i \in E^{c}(g)$. The first equality is due to the fact that each link in the network $\left.g\right|_{E^{c}(g)}$ is counted twice in the sum $\sum_{k \in E^{c}(g)} l_{k}\left(\left.g\right|_{E^{c}(g)}\right)$, and the second equality is trivial since the number of links in each network is simply the sum of own links and other players' links which is true for every player. Summing over all $i \in E^{c}(g)$ then yields

$$
\begin{aligned}
\sum_{i \in E^{c}(g)}\left(\sum_{k \in E^{c}(g)} l_{k}\left(\left.g\right|_{E^{c}(g)}\right)\right) & =2 \sum_{i \in E^{c}(g)} l_{i}\left(\left.g\right|_{E^{c}(g)}\right)+2 \sum_{i \in E^{c}(g)} l_{-i}\left(\left.g\right|_{E^{c}(g)}\right) \\
\Leftrightarrow \quad\left(\left|E^{c}(g)\right|-2\right)\left(\sum_{k \in E^{c}(g)} l_{k}\left(\left.g\right|_{E^{c}(g)}\right)\right) & =2 \sum_{k \in E^{c}(g)} l_{-k}\left(\left.g\right|_{E^{c}(g)}\right) .
\end{aligned}
$$

In order to complete the proof, we show that $E^{c}(g)$ must be the empty set. To the contrary, suppose that $\left|E^{c}(g)\right| \geq 4$. Hence (6.2) implies

$$
\sum_{k \in E^{c}(g)} l_{k}\left(\left.g\right|_{E^{c}(g)}\right) \leq \sum_{k \in E^{c}(g)} l_{-k}\left(\left.g\right|_{E^{c}(g)}\right) .
$$

Denote by $\tilde{l}$ the number of links connecting $E(g)$ and $E^{c}(g)$, i.e. $\tilde{l}:=\mid\{i j \in$ $\left.g \mid i \in E(g), j \in E^{c}(g)\right\} \mid$. Then, $\sum_{k \in E^{c}(g)} l_{k}(g)=\sum_{k \in E^{c}(g)} l_{k}\left(\left.g\right|_{E^{c}(g)}\right)+\tilde{l}$ and $\sum_{k \in E^{c}(g)} l_{-k}\left(\left.g\right|_{E^{c}(g)}\right)+\left(\left|E^{c}(g)\right|-1\right) \tilde{l} \leq \sum_{k \in E^{c}(g)} l_{-k}(g)$. Since $\left|E^{c}(g)\right| \geq 4$, we then get by $(6.3), \sum_{k \in E^{c}(g)} l_{k}(g) \leq \sum_{k \in E^{c}(g)} l_{-k}(g)$, contradicting that $l_{i}(g)>l_{-i}(g)$ for all $i \in E^{c}(g)$.

Finally consider $\left|E^{c}(g)\right| \leq 3$. Note that by assumption $n \geq 5$. If $\left|E^{c}(g)\right| \in\{1,2\}$, there is at most one link in $\left.g\right|_{E^{c}(g)}$ while $|E(g)| \geq 3$ and completely connected, implying that $l_{i}(g) \leq 1<3 \leq l_{-i}(g)$ for $i \in E^{c}(g)$, a contradiction. If $\left|E^{c}(g)\right|=3$ there are at most three links in $\left.g\right|_{E^{c}(g)}$. We hence have $\sum_{k \in E^{c}(g)} l_{k}\left(\left.g\right|_{E^{c}(g)}\right) \leq$ $\sum_{k \in E^{c}(g)} l_{-k}\left(\left.g\right|_{E^{c}(g)}\right)+3$. Since $n \geq 5$ and hence $|E(g)| \geq 2$ we have $|g|_{E(g)} \mid \geq 1$ and thus if there are no connections between $E(g)$ and $E\left(g^{c}\right), l_{-i}\left(\left.g\right|_{E^{c}(g)}\right)+1 \leq$ $l_{-i}(g)$ for all $i \in E^{c}(g)$. Denoting, as above, by $\tilde{l}$ the number of links connecting $E(g)$ and $E^{c}(g)$, we then get

$$
\sum_{k \in E^{c}(g)} l_{k}(g)=\tilde{l}+\sum_{k \in E^{c}(g)} l_{k}\left(\left.g\right|_{E^{c}(g)}\right) \leq 3+\tilde{l}+\sum_{k \in E^{c}(g)} l_{-k}\left(\left.g\right|_{E^{c}(g)}\right) \leq \sum_{k \in E^{c}(g)} l_{-k}(g),
$$


contradicting that $l_{i}(g)>l_{-i}(g)$ for all $i \in E^{c}(g)$. Thus $E^{c}(g)$ must be the empty set implying that $g=\left.g\right|_{E(g)}$ and hence must be completely connected to be pairwise stable.

The equivalent argument in case of $g^{N}$ not being deletion proof completes the proof.

Proof of Corollary 1. Let $g^{\emptyset}$ be not pairwise stable. Take now any $g \in G$. We need to show that $g$ is not PS unless $g=g^{N}$.

First, by anonymity of the utility profile we have $\Delta u_{i}\left(g^{\emptyset}+i j, i j\right)>0$ for all $i, j \in N$. Now, take the decomposition of $g$ into $L_{i}(g)$ (links of player $i$ ) and $L_{-i}(g)$ (all other links), and observe that

$$
\begin{aligned}
& \Delta u_{i}\left(g^{\emptyset}+i j, i j\right)>0 \\
\Rightarrow & \Delta u_{i}\left(g^{\emptyset}+L_{i}(g)+i j, i j\right)>0 \\
\Rightarrow & \Delta u_{i}\left(g^{\emptyset}+L_{i}(g)+L_{-i}(g)+i j, i j\right)>0 \\
\Rightarrow & \Delta u_{i}(g+i j, i j)>0,
\end{aligned}
$$

where the first implication holds by convexity and the second one by strategic complements. Thus, no network but the complete one can be addition proof.

Finally, as (6.4) holds also for $g=g^{N}-i j$ and for all $i, j \in N$, the complete network $g^{N}$ is deletion proof and thus pairwise stable.

The equivalent argument in case of $g^{N}$ not being deletion proof completes the proof.

Proof of Proposition 2. Suppose to the contrary that there exists a pairwise stable network which is not a nested split graph. Then by definition there exists a set of three distinct players $i, j, k$, such that $\eta_{i}(g) \geq \eta_{j}(g) \geq \eta_{k}(g)$, and either $i k \in g$ while $i j \notin g$ or $j k \in g$ while $i k \notin g$.

Suppose first $i k \in g, i j \notin g$. Since $g$ is assumed to be stable, we have $\Delta u_{i}(g, i k) \geq$ 0 and $\Delta u_{k}(g, i k) \geq 0$. Then however

$$
\Delta u_{i}(g, i k) \geq 0 \Rightarrow \Delta u_{i}(g+i j, i j)>0,
$$

following by SPC, and further

$$
\Delta u_{k}(g, i k) \geq 0 \Rightarrow \Delta u_{j}(g+i j, i j) \geq 0,
$$

following by anonymous convexity. Thus $i$ and $j$ would want to add a link to $g$, contradicting pairwise stability. 
If on the other hand $j k \in g, i k \notin g$ we can argue similarly

$$
\Delta u_{k}(g, j k) \geq 0 \Rightarrow \Delta u_{k}(g+i k, i k)>0,
$$

by SPC, and

$$
\Delta u_{j}(g, j k) \geq 0 \Rightarrow \Delta u_{i}(g+i k, i k) \geq 0
$$

by anonymous convexity. Again, $i$ and $k$ would want to add a link, so that $g$ cannot be stable.

Proof of Corollary 2. The idea is to find a threshold for which any terms of order $\delta^{3}$ and higher can be disregarded.

Remember that

$$
u_{i}^{B C}=b_{i}(g)-\eta_{i}(g) c=e_{i}^{\prime}\left[\sum_{t=0}^{\infty} \delta^{t} A^{t}\right] \overrightarrow{1}-\eta_{i}(g) c
$$

$A$ being the adjacency matrix of network $g$ and $e_{i}$ the $i$-th unit vector, and thus $\Delta u_{i}^{B C}(g+i j, i j)=b_{i}(g+i j)-b_{i}(g)-c=\delta+\delta^{2} \eta_{j}(g+i j)+e_{i}^{\prime}\left[\sum_{t=3}^{\infty} \delta^{t}\left(A_{+i j}^{t}-A^{t}\right)\right] \overrightarrow{1}-c$,

where $A_{+i j}$ is the adjacency matrix corresponding to the network $g+i j$.

Now, take some players $i, j, k \in N$ and a network $g$ such that $i j \in g, i k \notin g$ and $\eta_{j}(g) \leq \eta_{k}(g)$. We can find a lower bound for marginal utility of adding $k$ by disregarding third order terms,

$$
\Delta u_{i}^{B C}(g+i k, i k) \geq \delta+\delta^{2}\left(\eta_{k}(g)+1\right)-c,
$$

and we can find an upper bound for the marginal utility of deleting $j$ by considering utility of the complete network from order 3 on, ${ }^{19}$

$$
\begin{aligned}
\Delta u_{i}^{B C}(g, i j) & \leq \delta+\delta^{2} \eta_{j}(g)+\sum_{t=3}^{\infty} \delta^{t} \eta_{j}(g)(n-1)^{t-2}-c \\
& =\delta+\delta^{2} \eta_{j}(g)+\delta^{2} \eta_{j}(g) \sum_{t=1}^{\infty} \delta^{t}(n-1)^{t}-c
\end{aligned}
$$

\footnotetext{
${ }^{19}$ Notice that the approximations used are quite rough. For example, instead of using the empty network as a lower bound approximation, one could instead use the star network of $\eta_{k}(g)+1$ players.
} 


$$
\begin{aligned}
& =\delta+\delta^{2} \eta_{j}(g)+\delta^{2} \eta_{j}(g)\left(\sum_{t=0}^{\infty} \delta^{t}(n-1)^{t}-1\right)-c \\
& =\delta+\delta^{2} \eta_{j}(g)+\delta^{2} \eta_{j}(g)\left(\frac{1}{1-\delta(n-1)}-1\right)-c \\
& =\delta+\frac{\delta^{2} \eta_{j}(g)}{1-\delta(n-1)}-c .
\end{aligned}
$$

With this we get

$$
\begin{array}{ll}
\delta+\delta^{2}\left(\eta_{k}(g)+1\right)-c \geq \delta+\frac{\delta^{2} \eta_{j}(g)}{1-\delta(n-1)}-c & \forall \eta_{j}(g) \leq \eta_{k}(g) \\
\Leftrightarrow \quad \eta_{k}(g)+1 \geq \frac{\eta_{j}(g)}{1-\delta(n-1)} & \forall \eta_{j}(g) \leq \eta_{k}(g) \\
\Leftrightarrow \quad 1-\delta(n-1) \geq \frac{\eta_{j}(g)}{\eta_{k}(g)+1} & \forall \eta_{j}(g) \leq \eta_{k}(g)
\end{array}
$$

Since $\eta_{k}(g) \leq n-2$, the right-hand side is maximized for $\eta_{j}(g)=\eta_{k}(g)=n-2$. Thus,

$$
\begin{aligned}
& \Leftrightarrow \quad 1-\delta(n-1) \geq \frac{n-2}{n-1} \\
& \Leftrightarrow \quad \delta \leq \frac{1}{(n-1)^{2}} .
\end{aligned}
$$

Thus, for $\delta<\frac{1}{(n-1)^{2}}$ it holds true that

$$
\eta_{j}(g) \leq \eta_{k}(g) \Rightarrow \Delta u_{i}^{B C}(g, i j)<\Delta u_{i}^{B C}(g+i k, i k) .
$$

Let now $i, j, k \in N$ such that $\eta_{j}(g) \leq \eta_{k}(g)$ and $\Delta u_{i}^{B C}(g, i j) \geq 0$. Then it directly follows from (6.5) that

$$
\Delta u_{i}^{B C}(g+i k, i k)>0,
$$

such that $u^{B C}$ satisfies strong preference for centrality.

Letting on the other hand $\eta_{i}(g) \leq \eta_{j}(g)$ and $\Delta u_{i}^{B C}(g, i k) \geq 0$, it is

$$
\begin{aligned}
0 & \leq \Delta u_{i}^{B C}(g, i k) \\
& =\delta+\delta^{2} \eta_{k}(g)+e_{i}^{\prime}\left[\sum_{t=3}^{\infty} \delta^{t}\left(A^{t}-A_{-i k}^{t}\right)\right] \overrightarrow{1}-c \\
& <\delta+\delta^{2} \eta_{k}(g+j k)+e_{j}^{\prime}\left[\sum_{t=3}^{\infty} \delta^{t}\left(A_{+j k}^{t}-A^{t}\right)\right] \overrightarrow{1}-c \\
& =\Delta u_{j}^{B C}(g+j k, j k),
\end{aligned}
$$

thus $u^{B C}$ also satisfies anonymous convexity. 
Proof of Lemma 2. Divide the set of players into two equal groups and label the players in the groups as $\left(i_{1}^{1}, i_{2}^{1}, \ldots, i_{n / 2}^{1} ; i_{1}^{2}, i_{2}^{2}, \ldots, i_{n / 2}^{2}\right)$. To construct a symmetric regular network of degree $1 \leq k \leq n / 2$, connect each player $i_{m}^{j}$ with players $\left(i_{m}^{3-j}, \ldots, i_{m+k-1}^{3-j}\right)$, where player $i_{p}^{j}=i_{p-n / 2}^{j}$, so that for example $i_{n / 2+1}^{1}, i_{1}^{1}$ are two labels for the same player. It is clear that with this construction all players are in symmetric positions.

Labeling the networks above as $g_{k}^{r}$ for $1 \leq k \leq n / 2$, a symmetric regular network of degree $n / 2+1 \leq k \leq n-2$ can be constructed as $g_{k}^{r}=g^{N}-g_{n-k-1}^{r}$.

Finally, the empty and the complete network are trivially symmetric, what completes the argument.

Proof of Proposition 3. The empty network $g^{\emptyset}$ is either pairwise stable or not addition proof. In the first case the result is already established, so suppose the latter, that is (with homogeneity)

$$
\Delta u_{i}\left(g^{\emptyset}+i j, i j\right)>0 \quad \forall i, j \in N .
$$

With strategic complements, also $\Delta u_{i}\left(g_{1}^{r}, i j\right)>0$. Notice that $g_{1}^{r}$ is necessarily a symmetric network, so that with anonymity it is $u_{i}\left(g_{1}^{r}\right)=u_{j}\left(g_{1}^{r}\right)$ for any two players $i, j \in N$ and by the same argument $\Delta u_{i}\left(g_{1}^{r}, i j\right)>0 \quad \forall i j \in g_{1}^{r}$, making $g_{1}^{r}$ deletion proof.

Take now any symmetric regular network $g_{k}^{r}$ of degree $1 \leq k \leq n-2$ that is deletion proof. It is either addition proof and hence pairwise stable or it holds for some $i, j \in N, i j \notin g_{k}^{r}$ that

$$
\Delta u_{i}\left(g_{k}^{r}+i j, i j\right)>0,
$$

and as the utility profile is degree-based and $g_{k}^{r}$ is symmetric also

$$
\Delta u_{i}\left(g_{k}^{r}+i j, i j\right)>0 \quad \forall i j \in g^{N}-g_{k}^{r} .
$$

With strategic complements it holds again that in this case also $\Delta u_{i}\left(g_{k+1}^{r}, i j\right)>$ $0 \forall i j \in g_{k+1}^{r}$, for $g_{k+1}^{r}$ being again a symmetric regular network which exists and can be reached by Lemma 2. Notice in particular that by assumption, as the last link was added with positive marginal utility, all links of the player and hence all links in the network are of positive marginal utility, as again the utility profile is degree-based. So $g_{k+1}^{r}$ is again deletion proof.

If $k+1=n / 2$ and $g_{n / 2}^{r}$ is not addition proof, change to $g^{N}-g_{n / 2-1}^{r}$ and proceed as above. Observe that $n / 2=(n-1)-(n / 2-1)$ and thus $g^{N}-g_{n / 2-1}^{r}$, so for a degree-based utility profile it is $u\left(g_{n / 2}^{r}\right)=u\left(g^{N}-g_{n / 2-1}^{r}\right)$, and thus the network is again deletion proof.

The finiteness of the setting, and in particular the existence of a maximal degree $(n-1)$ completes the argument and existence is established. 


\section{Proposition 7.}

Suppose the degree-based profile of utility functions satisfies strategic complements, weak preference for centrality and anonymity. If the number of players $n$ is odd, then there exists a restricted regular network that is pairwise stable.

Proof of Proposition \%. The proof is structurally the same as of Proposition 3. Suppose in any restricted regular network $\tilde{g}=g_{m}^{r r}, 1 \leq m<n-1$ player $i$ is the isolated player, and suppose that $\tilde{g}_{-i}$ is a symmetric network. Observe that if the empty network is not addition proof then an isolated player has strictly positive marginal utility of any link in any network due to strategic complements.

Now, if $\tilde{g}$ is not addition proof, then either $\Delta u_{j}(\tilde{g}+j k, j k)>0$ for all $j, k \in N \backslash\{i\}$ or $\Delta u_{j}(\tilde{g}+i j, i j)>0$ and by WPC also $\Delta u_{j}(\tilde{g}+j k, j k)>0$ for all $j, k \in N \backslash\{i\}$. Hence we get to the network $g_{m+1}^{r r}$, where again $i$ is isolated and $\left(g_{m+1}^{r r}\right)_{-i}$ is symmetric, by the same arguments as in the proof of Proposition 3.

Suppose finally that $\tilde{g}$ is such that $g_{-i}$ is complete. Note that $\Delta u_{i}(\tilde{g}+i j, i j)>$ 0 for all $j \neq i$, thus if $\Delta u_{j}(\tilde{g}+i j, i j)<0$ then $\tilde{g}$ is pairwise stable, but if $\Delta u_{j}(\tilde{g}+i j, i j)>0$ then link $i j$ is formed.

Let now $\hat{g}$ be such that $\hat{g}=\tilde{g}+l_{i}$, where $1 \leq\left|l_{i}\right| \leq n-2$. Observe that $\hat{g} \supset g_{\left|l_{i}\right|}^{r r}$. As $g_{\left|l_{i}\right|}^{r r}$ was not pairwise stable, it is $\Delta u_{i}\left(g_{\left|l_{i}\right|}^{r r}+i j, i j\right)>0, \eta_{j}\left(g_{\left|l_{i}\right|}^{r r}\right) \neq 0$, and as $\eta_{i}\left(g_{\left|l_{i}\right|}^{r r}\right)=\left|l_{i}\right|=\eta_{i}(\hat{g})$ by strategic complements also $\Delta u_{i}(\hat{g}+i j, i j)>0$ for all $j \in g^{N}-\hat{g}$ and the complete network is pairwise stable.

Proof of Proposition 4. Suppose the complete network is not deletion proof, i.e. it is not PS (otherwise there is nothing to show). Then by anonymity,

$$
\Delta u_{i}\left(g^{N}, i j\right)<0 \quad \forall i, j \in N
$$

. Hence taking one player $i \in N$ and deleting all her links yields by convexity

$$
\Delta u_{i}\left(g^{N}-L_{i}\left(g^{N}\right)+i j, i j\right) \leq \Delta u_{i}\left(g^{N}-i j+i j, i j\right)<0 \quad \forall i, j \in N
$$

such that the network $g^{N}-L_{i}\left(g^{N}\right)$ which is the dominant group network $g_{n-1}^{d g}$ is addition proof.

Now let a dominant group network $g_{m}^{d g}$ of size $m$ be addition proof. If it is also deletion proof then it is pairwise stable in which case there is nothing more to show. Hence, suppose that $g_{m}^{d g}$ is not deletion proof. Then, there exists a player $i \in N$ such that $\Delta u_{i}\left(g_{m}^{d g}, i j\right)<0$.

Now, by convexity it is also $\Delta u_{i}\left(g_{m}^{d g}-l_{i}\left(g_{m}^{d g}\right)+i j, i j\right)<0$, where $l_{i}\left(g_{m}^{d g}\right)=\{i k \in$ $\left.g_{m}^{d g}\right\}$. Observing that $g_{m}^{d g}-l_{i}\left(g_{m}^{d g}\right)=g_{m-1}^{d g}$ we have by anonymity that no isolated player $i \in I\left(g_{m-1}^{d g}\right)$ wants to form a link to any player of the dominant group. What is more, as $\emptyset=N_{j} \subset N_{k}$ for any $j \in I\left(g_{m-1}^{d g}\right)$ and $k \in C\left(g_{m-1}^{d g}\right)$, by 
WPC also isolated players do not want to form links in $g_{m-1}^{d g}$. Thus, $g_{m-1}^{d g}$ is again addition proof. Hence if no network $g_{m}^{d g}$ is PS then the empty network is necessarily pairwise stable. By definition, the empty and the complete network are dominant group networks, where the latter is addition proof (since there are no more links that can be added). Thus, by induction either all dominant group networks are addition proof in which case the empty network is PS (since there are no more links that can be deleted) or there exists network of dominant group structure which is deletion and addition proof, i.e. PS.

\section{References}

Acemoglu, D., Carvalho, V. M., Ozdaglar, A., and Tahbaz-Salehi, A. (2012). The network origins of aggregate fluctuations. Econometrica, 80(5):1977-2016.

Allouch, N. (2012). On the private provision of public goods on networks. FEEM Working Paper No. 40.2012.

Ballester, C., Calvó-Armengol, A., and Zenou, Y. (2006). Who's who in networks. Wanted: The key player. Econometrica, 74(5):1403-1417.

Biggs, N. (1994). Algebraic graph theory. Cambridge University Press.

Bloch, F. (1997). Non-cooperative models of coalition formation in games with spillovers, chapter 10, pages 311-352. Cambridge University Press.

Bloch, F. and Jackson, M. O. (2006). Definitions of equilibrium in network formation games. International Journal of Game Theory, 34:305-318.

Bloch, F. and Jackson, M. O. (2007). The formation of networks with transfers among players. Journal of Economic Theory, 133(1):83-110.

Bonacich, P. (1987). Power and centrality: A family of measures. American Journal of Sociology, 92(5):1170-1182.

Bosker, M. and Westbrock, B. (2014). A theory of trade in a global production network. CEPR Discussion Paper No. DP9870.

Bramoullé, Y., Kranton, R., and D'Amours, M. (2014). Strategic interaction and networks. The American Economic Review, 104(3):898-930.

Calvó-Armengol, A. and Ilkiliç, R. (2009). Pairwise-stability and nash equilibria in network formation. International Journal of Game Theory, 38:51-79. 
Chakrabarti, S. and Gilles, R. (2007). Network potentials. Review of Economic Design, 11(1):13-52.

Cvetković, D. and Rowlinson, P. (1990). The largest eigenvalue of a graph: A survey. Linear and multilinear algebra, 28(1-2):3-33.

Dawid, H. and Hellmann, T. (2014). The evolution of r\&d networks. Journal of Economic Behavior \& Organization, 105:158-172.

Easly, D. and Kleinberg, J. (2010). Networks, Crowds and Markets - Reasoning about a Higly Connected World. Cambridge University Press.

Goyal, S. (2005). Learning in networks. In Demange, G. and Wooders, M., editors, Group Formation in Economics: Networks, Clubs and Coalitions, pages 122167. Cambridge University Press.

Goyal, S. (2007). Connections. Princeton University Press.

Goyal, S. and Joshi, S. (2003). Networks of collaboration in oligopoly. Games and Economic Behavior, 43(1):57 - 85.

Goyal, S. and Joshi, S. (2006a). Bilateralism and free trade. International Economic Review, 47(3):749-778.

Goyal, S. and Joshi, S. (2006b). Unequal connections. International Journal of Game Theory, 34:319-349.

Goyal, S. and Vega-Redondo, F. (2007). Structural holes in social networks. Journal of Economic Theory, 137(1):460-492.

Hellmann, T. (2013). On the existence and uniqueness of pairwise stable networks. International Journal of Game Theory, 42:211-237.

Hellmann, T. and Staudigl, M. (2014). Evolution of social networks. European Journal of Operational Research, 234(3):583-596.

Jackson, M. O. (2003). A Survey of Models of Network Formation: Stability and Efficiency. Cambridge University Press.

Jackson, M. O. (2006). The economics of social networks. In Blundell, R., Newey, W. K., and Persson, T., editors, Advances in Economics and Econometrics: Theory and Applications, Ninth World Congress, volume I. Cambridge University Press.

Jackson, M. O. (2008). Social and Economic Networks. Princeton University Press. 
Jackson, M. O. and Watts, A. (2001). The existence of pairwise stable networks. Seoul Journal of Economics, 14(3):299-321.

Jackson, M. O. and Wolinsky, A. (1996). A Strategic Model of Social and Economic Networks. Journal of Economic Theory, 71(1):44-74.

König, M. (2012). Network formation with local complements and global substitutes: The case of R\&D networks. Working paper.

König, M., Tessone, C. J., and Zenou, Y. (2014). Nestedness in networks: A theoretical model and some applications. Theoretical Economics, forthcoming.

Kranton, R. E. and Minehart, D. F. (2001). A Theory of Buyer-Seller Networks. American Economic Review, 91(3):485-508.

Mahadev, N. V. R. and Peled, U. N. (1995). Threshold Graphs and Related Topics. North Holland.

Monderer, D. and Shapley, L. (1996). Potential games. Games and Economic Behavior, 14:124-143.

Myerson, R. B. (1991). Game Theory: Analysis of Conflict. Harvard University Press.

Polanski, A. and Vega-Redondo, F. (2013). Markets, bargaining, and networks with heterogeneous agents. Working Paper University of East Anglia, Norwich, $U K$.

Roth, A. E. and Sotomayor, M. A. O. (1992). Two-sided matching: A study in game-theoretic modeling and analysis. Cambridge University Press.

Simić, S. K., Li Marzi, E. M., and Belardo, F. (2006). Connected graphs of fixed order and size with maximal index: Structural considerations. Le Matematiche, $59(1,2): 349-365$.

Vega-Redondo, F. (2007). Complex Social Networks. Cambridge University Press, Econometric Society Monograph series. 